\title{
THE NASA-UC ETA-EARTH PROGRAM. II. A PLANET ORBITING HD 156668 WITH A MINIMUM MASS OF FOUR EARTH MASSES*
}

\author{
Andrew W. Howard ${ }^{1,2,9}$, John Asher Johnson ${ }^{3}$, Geoffrey W. Marcy ${ }^{1}$, Debra A. Fischer ${ }^{4}$, Jason T. Wright $^{5}$, \\ Gregory W. Henry ${ }^{6}$, Howard IsaAcson ${ }^{1}$, JefF A. VAlenti ${ }^{7}$, Jay Anderson ${ }^{7}$, and Nikolai E. Piskunov ${ }^{8}$ \\ ${ }^{1}$ Department of Astronomy, University of California, Berkeley, CA 94720-3411, USA \\ ${ }^{2}$ Space Sciences Laboratory, University of California, Berkeley, CA 94720-7450, USA; howard@ astro.berkeley.edu \\ ${ }^{3}$ Department of Astrophysics, California Institute of Technology, MC 249-17, Pasadena, CA 91125, USA \\ ${ }^{4}$ Department of Astronomy, Yale University, New Haven, CT 06511, USA \\ ${ }^{5}$ Center for Exoplanets and Habitable Worlds, The Pennsylvania State University, University Park, PA 16802, USA \\ ${ }^{6}$ Center of Excellence in Information Systems, Tennessee State University, 3500 John A. Merritt Blvd., Box 9501, Nashville, TN 37209, USA \\ ${ }^{7}$ Space Telescope Science Institute, 3700 San Martin Dr., Baltimore, MD 21218, USA \\ ${ }^{8}$ Department of Astronomy and Space Physics, Uppsala University, Box 515, 75120 Uppsala, Sweden \\ Received 2010 March 16; accepted 2010 October 29; published 2010 December 16
}

\begin{abstract}
We report the discovery of HD $156668 \mathrm{~b}$, an extrasolar planet with a minimum mass of $M_{P} \sin i=4.15 M_{\oplus}$. This planet was discovered through Keplerian modeling of precise radial velocities from Keck-HIRES and is the second super-Earth to emerge from the NASA-UC Eta-Earth Survey. The best-fit orbit is consistent with circular and has a period of $P=4.6455$ days. The Doppler semi-amplitude of this planet, $K=1.89 \mathrm{~m} \mathrm{~s}^{-1}$, is among the lowest ever detected, on par with the detection of GJ 581 e using HARPS. A longer period $(P \approx 2.3$ years $)$, low-amplitude signal of unknown origin was also detected in the radial velocities and was filtered out of the data while fitting the short-period planet. Additional data are required to determine if the long-period signal is due to a second planet, stellar activity, or another source. Photometric observations using the Automated Photometric Telescopes at Fairborn Observatory show that HD 156668 (an old, quiet K3 dwarf) is photometrically constant over the radial velocity period to $0.1 \mathrm{mmag}$, supporting the existence of the planet. No transits were detected down to a photometric limit of $\sim 3 \mathrm{mmag}$, ruling out transiting planets dominated by extremely bloated atmospheres, but not precluding a transiting solid/liquid planet with a modest atmosphere.
\end{abstract}

Key words: planetary systems - stars: individual (HD 156668) - techniques: radial velocities

\section{INTRODUCTION}

The search for low-mass planets is driven by a desire to observationally study the full range of planetary systems in order to better understand their formation, dynamics, composition, and diversity. We also seek Earth-like worlds of terrestrial composition as a goal in itself and as targets for future transit and imaging observations. This search has taken several leaps forward recently because of instrumental improvements. The precision of radial velocity (RV) measurements with Keck-HIRES by the California Planet Survey group (Howard et al. 2009), HARPS (Lovis et al. 2006), and the AAT (O'Toole et al. 2009) has now reached $1 \mathrm{~m} \mathrm{~s}^{-1}$ or better and has led to the discovery of several super-Earths around nearby, bright stars. Ground-based transit surveys such as MEarth (Charbonneau et al. 2009) and HATNet (Bakos et al. 2010) have made important discoveries of transiting low-mass planets. Microlensing searches have detected two super-Earths orbiting distant stars (Beaulieu et al. 2006; Bennett et al. 2008) and the statistics of microlensing detections suggest than cold Neptunes are a factor of three more common that cold Jupiters (Sumi et al. 2010). From space, CoRoT has found a system with two super-Earths (one of which transits; Léger et al. 2009) and Kepler is poised to detect true Earth analogs in 1 AU orbits using transit photometry with a precision of $20 \mathrm{ppm}$ in $6.5 \mathrm{hr}$ (Borucki et al. 2009). In the next decade, SIM Lite (Unwin et al. 2008) will astrometrically

\footnotetext{
* Based on observations obtained at the W. M. Keck Observatory, which is operated jointly by the University of California and the California Institute of Technology. Keck time has been granted by both NASA and the University of California.

9 Townes Fellow.
}

characterize essentially all planets down to Earth mass orbiting $\sim 100$ nearby stars, as well as the more massive planets orbiting $\sim 1000$ stars

The Eta-Earth Survey plays a unique role in the study of low-mass exoplanets. The population of 230 GKM stars in the survey is nearly free of statistical bias since the stars were not chosen based on their likelihood of harboring a planet, but rather on proximity, brightness, and chromospheric activity. Each star is observed at least 20 times, insuring a minimum detectability threshold. Thus, the distributions of planet detections and non-detections from the Eta-Earth Survey will yield a wealth of information about the efficiency and mechanisms of planet formation as well as the range of subsequent dynamical histories. The 20 survey observations per Eta-Earth Survey target are nearly complete and we are aggressively re-observing several promising candidate lowmass planets.

Current theories of planet formation (Ida \& Lin 2004; Mordasini et al. 2009) are consistent with the measured distributions of massive planets (Saturn mass and above), but their predictions for the abundance and properties of low-mass planets are only now being observationally tested. In particular, they predict a dearth of planets below roughly Saturn mass in orbits inside the ice line. Such planets are predicted to be rare because once a planet core grows by planetesimal accretion to a threshold mass of $\sim 10 M_{\oplus}$, it undergoes runaway gas accretion and becomes a gas giant. While these theories consistently predict such a "planet desert," they differ in the contours of the desert in $M$ and $a$ as well as whether the planets below the critical core mass survive Type I inward migration in significant numbers. 
Table 1

Stellar Properties of HD 156668

\begin{tabular}{lc}
\hline \hline \multicolumn{1}{c}{ Parameter } & Value \\
\hline Spectral type & $\mathrm{K} 3 \mathrm{~V}$ \\
$M_{V}$ & 6.480 \\
$B-V$ & 1.015 \\
$V$ & 8.424 \\
$J$ & 6.593 \\
$H$ & 6.117 \\
$K$ & 6.004 \\
Distance $(\mathrm{pc})$ & $24.5 \pm 0.5$ \\
{$[\mathrm{Fe} / \mathrm{H}]$} & $+0.05 \pm 0.06$ \\
$T_{\text {eff }}(\mathrm{K})$ & $4850 \pm 88$ \\
$v$ sin $i\left(\mathrm{~km} \mathrm{~s}{ }^{-1}\right)$ & $0.50 \pm 1.0$ \\
$\log g$ & $4.598 \pm 0.12$ \\
$L_{\star}\left(L_{\odot}\right)$ & $0.230 \pm 0.018$ \\
$M_{\star}\left(M_{\odot}\right)$ & $0.772 \pm 0.020$ \\
$R_{\star}\left(R_{\odot}\right)$ & $0.720 \pm 0.013$ \\
Age $(\mathrm{Gyr})$ & $8.6 \pm 4.8$ \\
$\log R_{\mathrm{HK}}^{\prime}$ & -4.98 \\
$S_{\mathrm{HK}}$ & 0.23 \\
$P_{\text {rot }}($ days $)$ & 51.5 \\
$\sigma_{\text {phot }}(\mathrm{mag})$ & $\lesssim 0.002$ \\
\hline
\end{tabular}

In this context we announce the discovery of HD $156668 \mathrm{~b}$, a super-Earth planet with a minimum mass of $M_{P} \sin i=4.15 M_{\oplus}$ and an orbital period of $P=4.6455$ days. This is the second super-Earth $\left(M \sin i<10 M_{\oplus}\right)$ to emerge from Keck observations explicitly for the NASA-UC Eta-Earth Survey, the first being HD 7924 b (Howard et al. 2009). The remainder of this paper is structured as follows. We describe the host star properties in Section 2. The spectroscopic observations and their Doppler reduction are described in Section 3. In Section 4, we describe the detection of the $P=4.6455$ day orbit of HD $156668 \mathrm{~b}$ and the high-pass filtering of the RV data that was necessary to obtain good estimates of the orbital parameters. In Section 5, we carefully consider the null hypothesis - the non-existence of HD $156668 \mathrm{~b}$ - using $S_{\mathrm{HK}}$ measurements, photometric observations, and false alarm probability (FAP) analyses. We summarize and discuss the implications of this discovery in Section 6.

\section{STELLAR PROPERTIES}

HD 156668 (HIP 84607) is a K3 dwarf (Gray et al. 2003) whose properties are summarized in Table 1. It is nearby ( $d=24.5$ pc; van Leeuwen 2007) and relatively bright $\left(V=8.424\right.$; Høg et al. 2000). With $M_{V}=6.480$ and $B-V=$ 1.015 , the star lies near the Hipparcos average main sequence as defined by Wright (2005).

Using the Spectroscopy Made Easy (SME) LTE spectral synthesis code (Valenti \& Fischer 2005), we analyzed two high-resolution, iodine-free Keck-HIRES spectra of HD 156668 and found the effective temperature, surface gravity, projected rotational velocity, and iron abundance ratio listed in Table 1. The errors on these four quantities have been conservatively doubled from the formal SME parameter uncertainties because the stellar atmosphere models are less certain at low $T_{\text {eff }}$ (the SME catalog; Valenti \& Fischer 2005 models stars down to $\left.T_{\text {eff }}=4800 \mathrm{~K}\right)$. We refined the above parameters and derived stellar mass, radius, and luminosity from SME and interpolated Yonsei-Yale ( $\mathrm{Y}^{2}$ ) isochrones (Demarque et al. 2004) using an iterative process that self-consistently ties together the SME and $\mathrm{Y}^{2}$ values of $\log g$ (Valenti et al. 2009). HD 156668 appears to be a typical middle-aged $\mathrm{K}$ dwarf. Its slightly super-solar iron

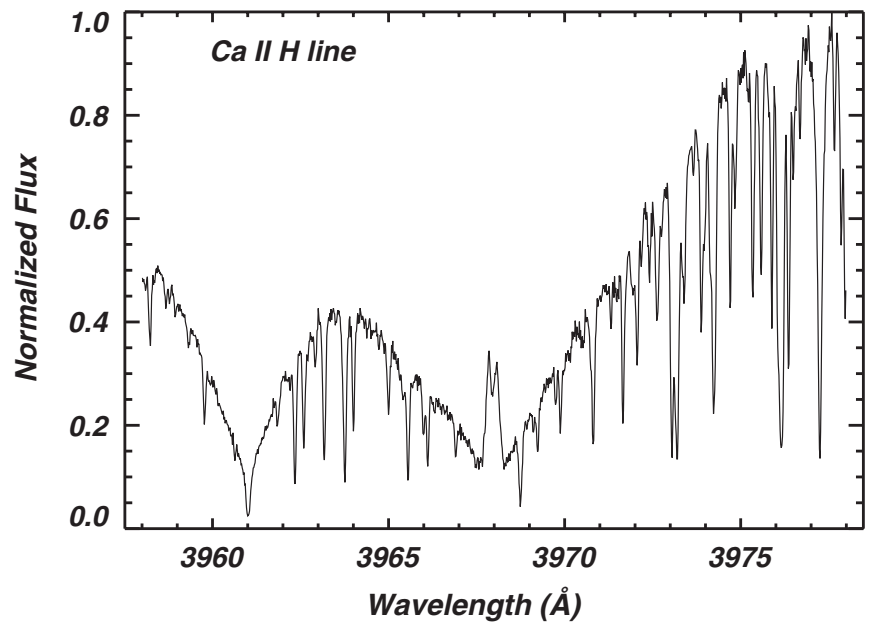

Figure 1. Ca II H line for HD 156668 from a Keck/HIRES spectrum. The line core emission near $3968 \AA$ indicates modest chromospheric activity.

abundance of $[\mathrm{Fe} / \mathrm{H}]=+0.05 \pm 0.06$ is consistent with its location near the average main sequence.

Measurements of the cores of the $\mathrm{Ca}$ II $\mathrm{H}$ and $\mathrm{K}$ lines show that HD 156668 has modest chromospheric activity (Figure 1). We measured the chromospheric activity indices $S_{\mathrm{HK}}=0.23$ and $\log R_{\mathrm{HK}}^{\prime}=-4.98$ using the method described Wright et al. (2004) and Isaacson \& Fischer (2010). The full set of $S_{\mathrm{HK}}$ measurements for all observations of HD 156668 does not show a periodicity near the planet's orbital of 4.6455 days (see Section 5.3 for additional discussion).

We estimate a rotation period $P_{\mathrm{rot}} \sim 48$ days using $R_{\mathrm{HK}}^{\prime}$ and $B-V$ calibration (Noyes et al. 1984), which is consistent with the 51.5 day period measured by automatic photometric telescope (APT) photometry (see Section 5.2). Following Wright (2005), and based on the values of $S_{\mathrm{HK}}, M_{V}$, and $B-V$, we estimate an RV jitter of $1.5 \mathrm{~m} \mathrm{~s}^{-1}$. This empirical estimate is based on an ensemble of stars with similar characteristics and accounts for RV variability due to rotational modulation of stellar surface features, stellar pulsation, undetected planets, and uncorrected systematic errors in the velocity reduction (Saar et al. 1998; Wright 2005). As explained in Section 4, jitter is added in quadrature to the RV measurement uncertainties for Keplerian fitting.

HD 156668 has several important characteristics that make it a nearly ideal RV target star. Like other old, chromospherically quiet stars with spectral types from late $\mathrm{G}$ to early $\mathrm{K}$, HD 156668 appears to be near the minimum of astrophysical jitter arising from acoustic oscillations, granulation, and photospheric activity (see Section 5.3 and Figure 7). The star is relatively bright, yielding high signal-to-noise spectra in 4-5 minutes per observation.

\section{HIRES OBSERVATIONS AND DOPPLER REDUCTION}

We observed HD 156668 with the HIRES echelle spectrometer (Vogt et al. 1994) on the $10 \mathrm{~m}$ Keck I telescope. The 107 observations span five years (2005-2009) with high-cadence observations - clusters of observations on 6-12 consecutive nights-beginning in 2007. All observations were made with an iodine cell mounted directly in front of the spectrometer entrance slit. The dense set of molecular absorption lines imprinted on the stellar spectra provides a robust wavelength fiducial against which Doppler shifts are measured, as well as strong constraints on the shape of the spectrometer instrumental profile at the 


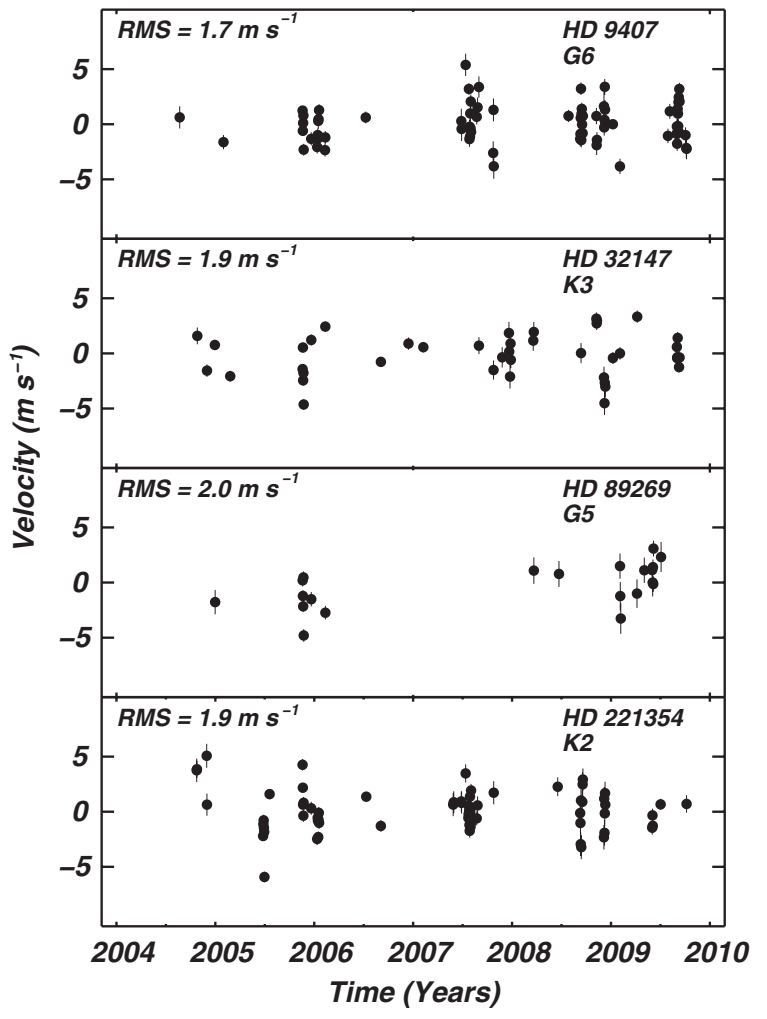

Figure 2. Radial velocity time series for four stable stars in our Keck Doppler survey that are similar to HD 156668. These stars demonstrate longterm velocity stability and precision of HIRES. The binned velocities with measurement uncertainties (but not jitter) are plotted. Panels are labeled with star name, spectral type, and velocity rms.

time of each observation (Marcy \& Butler 1992; Valenti et al. 1995).

We measured the Doppler shift from each star-times-iodine spectrum using a modeling procedure modified from the method described by Butler et al. (1996). The most significant modification is the way we model the intrinsic stellar spectrum, which serves as a reference point for the relative Doppler shift measurements for each observation. Butler et al. use a version of the Jansson (1995) deconvolution algorithm to remove the spectrometer's instrumental profile from an iodine-free template spectrum. We instead use a new deconvolution algorithm developed by one of us (J.A.J.) that employs a more effective regularization scheme, which results in significantly less noise amplification and improved Doppler precision.

Figure 2 shows RV time series for four stable stars with characteristics similar to HD 156668, demonstrating our measurement precision of $\sim 1.5-2.0 \mathrm{~m} \mathrm{~s}^{-1}$ (including astrophysical, instrumental/systematic, and photon-limited errors) for chromospherically quiet late $\mathrm{G} /$ early $\mathrm{K}$ stars over the past five years. All of the measurements reported here were made after the HIRES CCD upgrade in 2004 August and do not suffer from the higher noise and systematic errors that limited the precision of pre-upgrade measurements to $\sim 2-3 \mathrm{~m} \mathrm{~s}^{-1}$ for most stars.

The velocities derived from the 107 observations have a median single measurement uncertainty of $1.01 \mathrm{~m} \mathrm{~s}^{-1}$. This uncertainty is the weighted standard deviation of the mean of the velocity measured from each of the $\sim 7002 \AA$ chunks in each echelle spectrum (Butler et al. 1996). In a few cases, we made consecutive observations of HD 156668 to reduce the Poisson noise from photon statistics. For the Keplerian orbital analysis below (Section 4), the velocities were binned in $2 \mathrm{hr}$ intervals, yielding 86 measurements with an rms of $2.71 \mathrm{~m} \mathrm{~s}^{-1}$ about the mean and a median measurement uncertainty of $0.99 \mathrm{~m} \mathrm{~s}^{-1}$.

\section{ORBITAL ANALYSIS}

The measured radial velocities of HD 156668 are listed in Table 2 and plotted as a time series in Figure 3(a). Before any fitting or filtering, these velocities already have a low rms, $\sigma=2.71 \mathrm{~m} \mathrm{~s}^{-1}$. However, long-term trends, coherent over at least several months, are apparent by visual inspection of Figure 3(a) (see, e.g., the downward trend in 2007). These trends manifest themselves in a Lomb-Scargle periodogram (Lomb 1976; Scargle 1982) of the data as long-period power concentrated near $\sim 2.3$ years (Figure $3(\mathrm{e})$ ).

The RVs also show a significant periodicity near 4.647 days with power than exceeds the $0.1 \%$ FAP threshold. ${ }^{10}$ A second short-period peak is visible at 1.270 days, but this peak is a stroboscopic alias of the 4.647 day signal with the sidereal day length (see Section 5.4). We attempted to fit the radial velocities with a single-planet Keplerian orbital solution seeded with $P=4.647$ days using a partially linearized, least-squares fitting procedure (Wright \& Howard 2009). Each velocity measurement was assigned a weight constructed from the quadrature sum of the measurement uncertainty (listed in Table 2) and a stellar jitter term $\left(1.5 \mathrm{~m} \mathrm{~s}^{-1}\right)$. The fitting routine converged on a robust solution with $P=4.6455$ days, $e=0.39$, and $K=2.28 \mathrm{~m} \mathrm{~s}^{-1}$. This model gives $\sigma=2.33 \mathrm{~m} \mathrm{~s}^{-1}$ and $\chi_{v}=1.70$, a significant improvement over a linear model to the data.

We also tried fitting the long-period signal with a singleplanet Keplerian model. We seeded the fitting routine with a range of periods and found a best-fit solution with $P=791$ days (2.17 years) and a poorly constrained eccentricity when that parameter is allowed to float. The improvements in $\sigma$ and $\chi_{v}$ for this model are comparable to those from the $P=4.6455$ day model described above. However, the poorly constrained and high eccentricity of the fit and the uneven phase coverage render the interpretation of this signal suspect. We cannot be sure if it is due to a planet in Keplerian motion, an astrophysical signal such as chromospheric activity masquerading as an RV change of the star, or some other effect.

Whatever the source of the long-period signal, its effect is to obscure the short-period signal. We considered several ways to high-pass filter the RVs to isolate the short-period signal. A key requirement of such a filtering process is that it leave the short-period signal untouched. We concluded that fitting the data to a two-planet model plus a linear trend would robustly separate the long- and short-period signals, allowing for accurate parameter estimation for the short-period signal and a fair assessment of its statistical significance. The fitting routine converged on a robust solution with Keplerian parameters for the inner planet listed in Table 3. We adopt these parameters for HD $156668 \mathrm{~b}$. The "outer planet" in this model has $P=2.31$ years, $e=0.48, K=4.05 \mathrm{~m} \mathrm{~s}^{-1}, \omega=178^{\circ}$, $T_{\mathrm{p}}=2,454,414.22$, and $d v / d t=-0.94 \mathrm{~m} \mathrm{~s}^{-1} \mathrm{yr}^{-1}$ as shown by the dashed line in Figure 3. We emphasize that the outer planet in this fit is only a convenient model for the data, effectively

\footnotetext{
10 The false alarm probability (FAP) thresholds plotted as dashed horizontal lines in the periodograms in Figure 3 refer to the probability that periodograms of random rearrangements of the data would exceed the specified power level. Since periodograms only measure the power of sine wave fits to the data (i.e., circular orbits), these FAPS are less conservative than the ones described in Section 5.1 that allow for eccentric orbits and use the $\Delta \chi_{v}^{2}$ statistic.
} 
Table 2

Radial Velocities and $S_{\mathrm{HK}}$ Values for HD 156668

\begin{tabular}{|c|c|c|c|}
\hline JD - 2440000 & $\begin{array}{l}\text { Radial Velocity } \\
\qquad\left(\mathrm{m} \mathrm{s}^{-1}\right)\end{array}$ & $\begin{array}{l}\text { Uncertainty } \\
\left(\mathrm{m} \mathrm{s}^{-1}\right)\end{array}$ & $S_{\mathrm{HK}}$ \\
\hline 13478.97768 & -1.04 & 1.03 & 0.202 \\
\hline 13547.90964 & -4.86 & 0.99 & 0.214 \\
\hline 13604.83890 & -5.21 & 0.85 & 0.213 \\
\hline 13807.14411 & 3.29 & 1.14 & 0.222 \\
\hline 13932.91866 & 0.87 & 1.00 & 0.229 \\
\hline 13960.91401 & 3.29 & 0.87 & 0.220 \\
\hline 13961.80956 & 0.06 & 0.88 & 0.219 \\
\hline 13981.77064 & 3.87 & 0.94 & 0.231 \\
\hline 13982.87659 & 0.94 & 0.84 & 0.233 \\
\hline 13983.81911 & 4.28 & 0.80 & 0.230 \\
\hline 13984.90602 & 5.74 & 0.85 & 0.231 \\
\hline 14247.01977 & 1.27 & 0.71 & 0.239 \\
\hline 14248.00711 & 1.47 & 1.10 & 0.238 \\
\hline 14249.92154 & 0.79 & 1.14 & 0.235 \\
\hline 14252.01612 & 4.28 & 1.08 & 0.234 \\
\hline 14255.84743 & -0.27 & 0.76 & 0.238 \\
\hline 14277.79111 & -3.66 & 1.11 & 0.230 \\
\hline 14278.80991 & -2.37 & 0.98 & 0.230 \\
\hline 14285.81562 & 0.75 & 1.10 & 0.230 \\
\hline 14294.89361 & 2.08 & 1.16 & 0.226 \\
\hline 14304.95027 & -0.99 & 0.75 & 0.232 \\
\hline 14305.95179 & -2.31 & 0.69 & 0.234 \\
\hline 14306.92739 & -2.31 & 0.70 & 0.232 \\
\hline 14307.97764 & -0.33 & 0.73 & 0.231 \\
\hline 14308.94701 & 2.07 & 1.00 & 0.229 \\
\hline 14309.94163 & 0.68 & 0.71 & 0.232 \\
\hline 14310.93496 & 0.77 & 0.66 & 0.230 \\
\hline 14311.92755 & -1.40 & 0.67 & 0.230 \\
\hline 14312.93048 & 1.49 & 0.72 & 0.228 \\
\hline 14313.92787 & 2.74 & 0.68 & 0.228 \\
\hline 14314.96879 & -2.10 & 1.01 & 0.227 \\
\hline 14335.87372 & -2.54 & 0.63 & 0.229 \\
\hline 14343.79865 & -4.20 & 0.89 & 0.219 \\
\hline 14396.70306 & -3.13 & 0.95 & 0.221 \\
\hline 14397.70527 & -4.94 & 0.93 & 0.222 \\
\hline 14398.73491 & -6.92 & 1.11 & 0.219 \\
\hline 14636.95177 & -3.36 & 1.23 & 0.238 \\
\hline 14717.74461 & 3.34 & 0.99 & 0.254 \\
\hline 14718.89500 & -0.22 & 0.97 & 0.252 \\
\hline 14719.78911 & -4.40 & 1.02 & 0.256 \\
\hline 14720.82821 & 2.28 & 0.92 & 0.255 \\
\hline 14721.81321 & 5.53 & 1.00 & 0.255 \\
\hline 14722.75844 & 0.73 & 0.97 & 0.255 \\
\hline 14723.75113 & -0.66 & 0.90 & 0.255 \\
\hline 14724.77287 & -2.25 & 0.96 & 0.254 \\
\hline 14725.74632 & 0.03 & 1.03 & 0.252 \\
\hline 14726.80657 & 4.30 & 0.97 & 0.250 \\
\hline 14727.81096 & 1.82 & 0.92 & 0.250 \\
\hline 14777.68651 & 2.56 & 1.05 & 0.236 \\
\hline 14779.69190 & -4.18 & 1.23 & 0.235 \\
\hline 14927.05569 & -1.01 & 1.31 & 0.251 \\
\hline 14930.09564 & 2.92 & 1.26 & 0.244 \\
\hline 14935.05529 & 4.30 & 1.19 & 0.237 \\
\hline 14955.10678 & 4.61 & 1.16 & 0.239 \\
\hline 14955.97159 & 0.56 & 1.14 & 0.241 \\
\hline 14957.05565 & -1.73 & 1.05 & 0.241 \\
\hline 14983.97707 & -0.72 & 1.09 & 0.241 \\
\hline 14984.96665 & -2.64 & 1.22 & 0.242 \\
\hline 14985.92186 & 0.69 & 1.13 & 0.239 \\
\hline 14986.99173 & 1.99 & 1.14 & 0.240 \\
\hline 14987.99574 & -1.07 & 1.20 & 0.242 \\
\hline 14988.89618 & -2.23 & 1.08 & 0.244 \\
\hline 15016.97485 & -4.08 & 1.08 & 0.253 \\
\hline 15019.04148 & 0.23 & 1.25 & 0.252 \\
\hline 15041.97022 & -0.80 & 1.16 & 0.236 \\
\hline
\end{tabular}

Table 2

(Continued)

\begin{tabular}{lccc}
\hline \hline JD -2440000 & $\begin{array}{c}\text { Radial Velocity } \\
\left(\mathrm{m} \mathrm{s}^{-1}\right)\end{array}$ & $\begin{array}{c}\text { Uncertainty } \\
\left(\mathrm{m} \mathrm{s}^{-1}\right)\end{array}$ & $S_{\mathrm{HK}}$ \\
\hline 15042.89409 & 2.71 & 1.24 & 0.235 \\
15043.90466 & -1.71 & 1.13 & 0.233 \\
15044.94394 & -2.75 & 1.00 & 0.233 \\
15048.86672 & -2.97 & 1.26 & 0.239 \\
15073.76736 & -1.01 & 0.95 & 0.255 \\
15074.75962 & 1.61 & 0.91 & 0.253 \\
15075.73804 & 0.05 & 0.66 & 0.252 \\
15076.74458 & -2.42 & 0.68 & 0.247 \\
15077.74489 & -1.57 & 0.96 & 0.242 \\
15078.76561 & -2.64 & 1.00 & 0.239 \\
15079.73946 & -2.60 & 0.72 & 0.237 \\
15080.74369 & -0.13 & 0.69 & 0.235 \\
15081.73701 & -3.35 & 0.69 & 0.234 \\
15082.72821 & 0.83 & 1.01 & 0.229 \\
15083.73498 & 1.02 & 0.69 & 0.230 \\
15084.74428 & 1.19 & 1.00 & 0.228 \\
15106.76608 & -2.26 & 1.16 & 0.256 \\
15109.75425 & -3.25 & 1.39 & 0.261 \\
15111.72700 & -1.94 & 0.79 & 0.263 \\
15134.69674 & -0.50 & 0.82 & 0.232 \\
15135.70259 & -1.59 & 0.80 & 0.229 \\
\hline
\end{tabular}

Table 3

Orbital Solution for HD 156668 b

\begin{tabular}{lc}
\hline \hline \multicolumn{1}{c}{ Parameter } & Value \\
\hline$P($ days $)$ & $4.6455 \pm 0.0011$ \\
$T_{c}(\mathrm{JD}-2,440,000)$ & $14718.57 \pm 0.11$ \\
$e$ & $\equiv 0.0$ \\
$K\left(\mathrm{~m} \mathrm{~s}^{-1}\right)$ & $1.89 \pm 0.26$ \\
$M$ sin $i\left(M_{\oplus}\right)$ & $4.15 \pm 0.58$ \\
$a(\mathrm{AU})$ & $0.0500 \pm 0.0007$ \\
$N_{\text {obs }}($ binned $)$ & 86 \\
Median binned uncertainty $\left(\mathrm{m} \mathrm{s}^{-1}\right)$ & 0.99 \\
Assumed jitter $\left(\mathrm{m} \mathrm{s}^{-1}\right)$ & 1.50 \\
$\sigma\left(\mathrm{m} \mathrm{s}^{-1}\right)$ & 1.74 \\
$\sqrt{\chi_{v}^{2}}$ & 0.97 \\
\hline
\end{tabular}

serving as a high-pass filter to isolate the signal of the inner planet. Determining whether the long-period signal represents a planet will require additional RV measurements and diagnostic data.

Figure 3(b)/(f) shows the RV measurements after subtracting the long-period signal. Trends in the time series are no longer apparent and the 4.647 day signal and its alias at 1.270 days are significantly strengthened in the periodogram. Figure $3(\mathrm{c}) /(\mathrm{g})$ shows the RVs after subtracting both long- and short-period signals. The value of $\sigma$ is $1.74 \mathrm{~m} \mathrm{~s}^{-1}$ and the periodogram appears nearly featureless, with no periods remaining having significant power.

The phased orbital solution for HD $156668 \mathrm{~b}$ is shown in Figure 4. The Doppler semi-amplitude of $K=1.89 \mathrm{~m} \mathrm{~s}^{-1}$ is extremely low and is nearly equal to the $1.80 \mathrm{~m} \mathrm{~s}^{-1}$ typical error for single measurements (including jitter). The resulting minimum mass of $M \sin i=4.15 M_{\oplus}$ is also extremely small, and is the second lowest reported to date using the RV technique.

We adopted a circular orbit because the uncertainty on the eccentricity $(e=0.20 \pm 0.17)$ is significant when that parameter is allowed to float in the two-planet fit. Further, the $\chi^{2}$ difference between two-planet models with eccentric and circular inner planets $\left(\Delta \chi^{2}=1.3\right)$ is insufficient to statistically justify the 

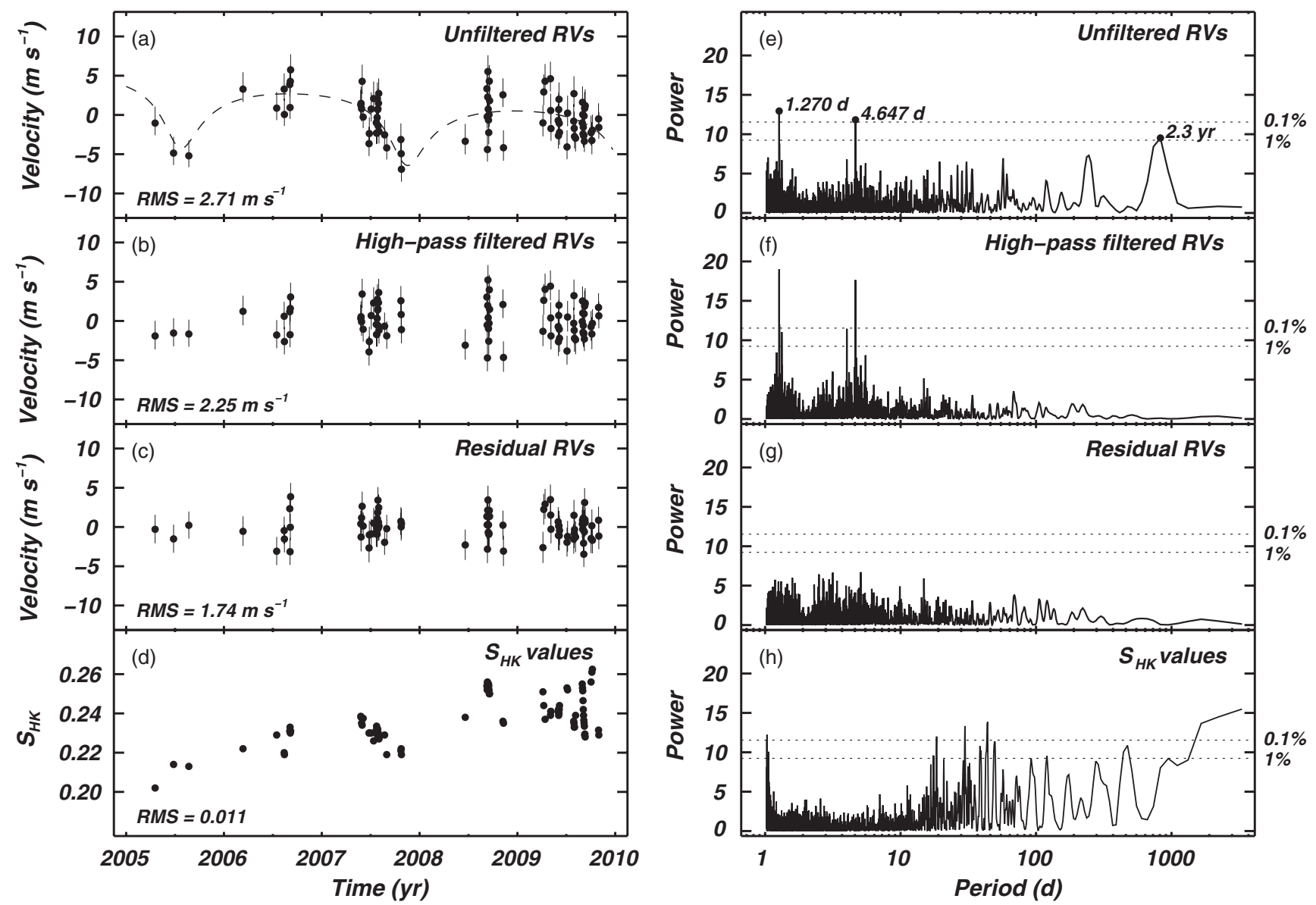

Figure 3. RV measurements and $S_{\mathrm{HK}}$ values for HD 156668 based on Keck-HIRES spectra. Each pair of panels in a given row displays the same data as a time series (panels (a)-(d), on left) and as a periodogram (panels (e)-(g), on right). Panels (a) and (e) show the unfiltered RVs from Table 2 with significant Fourier power at $\sim 2.3$ years, 4.647 days, and 1.270 days (an alias of 4.647 days with the sidereal day length). Panels (b) and (f) show the RVs after applying a high-pass filter by subtracting the dashed line model in panel (a); note that the $\sim 2.3$ year signal was almost completely removed, strengthening the power at 4.647 days and its alias. Panels (c) and (g) show the residuals of the high-pass filtered data to a one-planet fit with $P=4.6455$ days in a circular orbit. Panels (d) and (h) show values of the chromospheric index $S_{\mathrm{HK}}$ derived from each $\mathrm{RV}$ measurement.

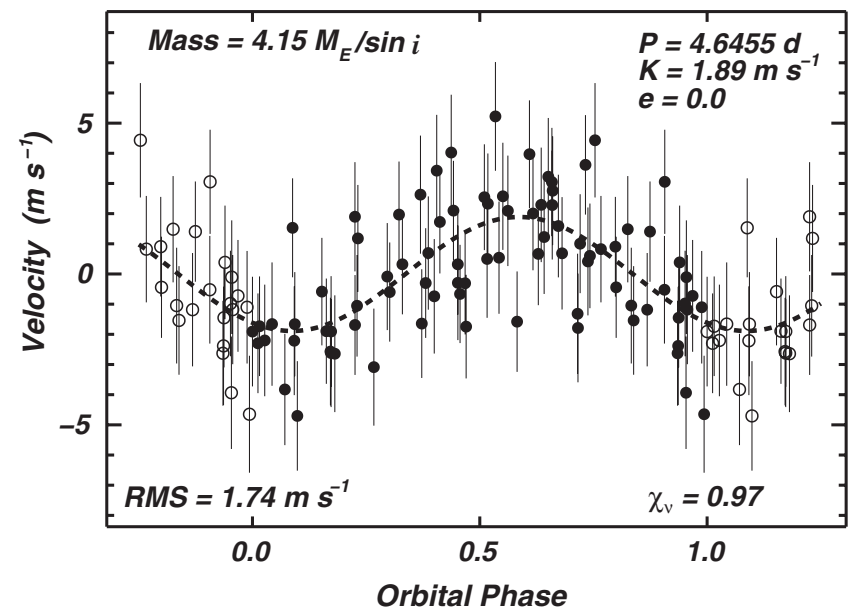

Figure 4. Single-planet model for the radial velocities of HD 156668, as measured by Keck-HIRES. The dashed line shows the best-fit circular orbital solution representing a $4.15 M_{\oplus}$ (minimum mass) planet in a 4.6455 day orbit. Filled circles represent phased, binned, and high-pass filtered velocities, while the open circles represent the same velocities wrapped one orbital phase. The error bars show the quadrature sum of measurement uncertainties and $1.5 \mathrm{~m} \mathrm{~s}^{-1}$ jitter.

more complicated eccentric model with two additional model parameters.
The Keplerian parameter uncertainties were derived using a Monte Carlo method (Marcy et al. 2005). The uncertainty estimates on $M \sin i$ and $a$ account for the uncertainty from $M_{\star}$. With five years of observations of this short-period planet, the error on $P$ is quite small (one part in 4000).

\section{THE NULL HYPOTHESIS}

In this section, we explicitly consider the null hypothesis. That is, we consider whether the $P=4.6455$ day signal we identified as due to a low-mass planet in Keplerian motion could be due to another source. Such an analysis is motivated by the extremely low amplitude signal $\left(K=1.89 \mathrm{~m} \mathrm{~s}^{-1}\right)$ and because of the use of high-pass filtering.

\subsection{False Alarm Probabilities}

We considered the possibility that the $P=4.6455$ day signal arose from the chance arrangement of random, statistically independent errors in the RVs by computing FAPs for several fits to the data (Marcy et al. 2005; Cumming 2004; Howard et al. 2009, 2010). These FAPs compare the measured data to 1000 scrambled data sets drawn randomly with replacement from unscrambled data. For each data set we compare a best-fit Keplerian model to the null hypothesis (a linear fit to the data) by computing $\Delta \chi_{v}^{2}=\chi_{\operatorname{lin}, v}^{2}-\chi_{\mathrm{Kep}, v}^{2}$, where $\chi_{\operatorname{lin}, v}^{2}$ and $\chi_{\mathrm{Kep}, v}^{2}$ are 
the values of $\chi_{\nu}^{2}$ for linear and Keplerian fits to the data, respectively. The $\Delta \chi_{v}^{2}$ statistic measures the improvement in the fit of a Keplerian model compared to a linear model of the same data. The FAP is the fraction of scrambled data sets that have a larger value of $\Delta \chi_{v}^{2}$ than for the unscrambled data set. That is, the FAP measures the fraction of scrambled data sets where the improvement in $\Delta \chi_{v}^{2}$ from a best-fit Keplerian model over a linear model is greater than the improvement of a Keplerian model over a linear model for the actual measured velocities. We use $\Delta \chi_{v}^{2}$ as the goodness-of-fit statistic, instead of other measures such as $\chi_{v}$ for a Keplerian fit, to account for the fact that the scrambled data sets, drawn from the original velocities with replacement, have different variances, which sometimes artificially improve the fit quality (i.e., some scrambled data sets contain fewer outlier velocities and have lower rms). Note that this FAP does not measure the probability of non-planetary sources of true velocity variation masquerading as a planetary signature.

We computed an FAP for the "two-planet model" (with the long-period planet acting as a high-pass filter) by comparing $\Delta \chi_{v}^{2}$ for the actual data with $\Delta \chi_{v}^{2}$ for two-planet fits to scrambled sets of the high-pass filtered data with the unscrambled longperiod signal added back in. This FAP has a value of $0.3 \%$ $(3 / 1000)$ and tests whether the statistical significance of the short-period signal is somehow enhanced by simultaneously fitting for a long-period signal.

We also computed the FAP for a one-planet fit to the high-pass filtered data. We compared $\Delta \chi_{v}^{2}$ for the unscrambled, filtered data set and scrambled versions thereof. This FAP tests whether the short-period signal is a statistical fluctuation, while assuming that the long-period signal is real (but still of unknown origin). We found an FAP of $0.4 \%$ (4/1000) for this scenario (with eccentricity unrestricted in the fits to scrambled data sets). We note that all four of the false alarm solutions had best-fit parameters that appeared unphysical: high eccentricity $(e>0.5)$ and short period $(P<10$ days). (We regard them as unphysical because short-period planets are almost universally in near circular orbits.) Such spurious, high-eccentricity solutions often appear as the best-fit solution to low rms RV data lacking a coherent signal. When we restricted the fits of scrambled data sets to circular orbits (as we did with the actual measurements), the FAP dropped to $<0.1 \%(0 / 1000)$.

We conclude that the $P=4.6455$ day signal is statistically significant. Considering all of the FAP tests, we estimate that the probability that this signal arose just due to random errors is less than $1 \%$ and probably on the order of $0.1 \%$.

It is worth noting that the horizontal dashed lines in Figures 3(e)-(h) represent analytic FAPs for uncorrelated Gaussian-distributed noise in a Lomb-Scargle periodogram (Scargle 1982). These analytic FAPs underestimate the true rate of false alarms for at least two reasons. First, by subtracting off the long-period component of our two-planet model (as depicted in the transition between panels (e) and (f) of Figure 3, which decreases the FAP from $\sim 0.1$ to $\ll 0.1$ ), we artificially boost the significance of the short-period signal. Second, the periodogram-based FAPs implicitly restrict the orbital search to circular orbits, thereby decreasing the rate of false alarms from eccentric solutions. Our estimate of the FAP described above accounts for these shortcomings in the periodogram-based FAP.

\subsection{Photometric Observations}

In addition to the Keck RVs, we obtained contemporaneous Strömgren $b$ and $y$ photometric measurements with the T10 $0.80 \mathrm{~m}$ APT at Fairborn Observatory in Arizona. This APT is
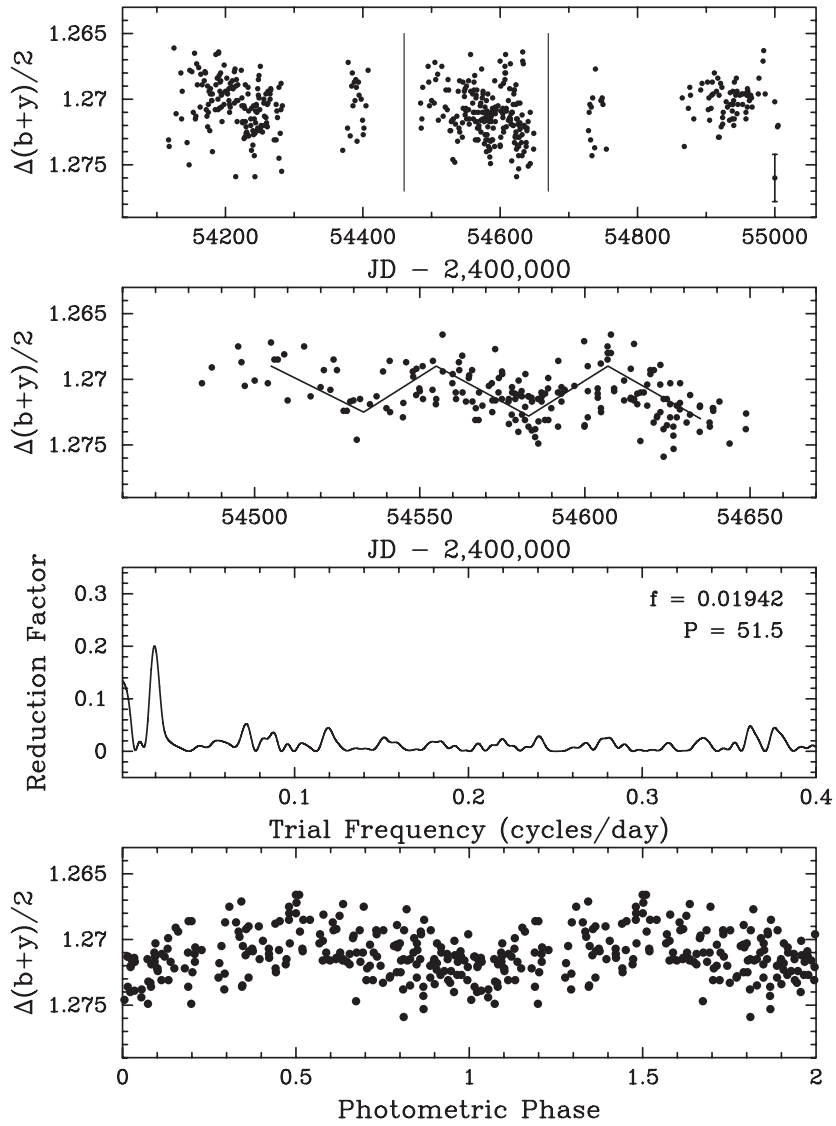

Figure 5. Top: the $477 D-(B+C) / 2$ photometric observations of HD 156668 in the $(b+y) / 2$ passband, acquired with the T10 $0.8 \mathrm{~m}$ APT during the 2007, 2008 , and 2009 observing seasons. Second panel: the portion of the 2008 observing season set off by vertical bars in the top panel shows the most coherent brightness variability due to cool star spots carried across the disk of the star by its rotation. Third panel: frequency spectrum of the observations in the second panel gives a best period of 51.5 days. Bottom panel: plot of the data from panel two phased with the 51.5 day period reveals coherent variability with a peak-to-peak brightness amplitude $0.0023 \mathrm{mag}$.

identical to the T8 APT that was used to acquire photometric observations of HD 7924 in the first paper of this series (Howard et al. 2009). (That paper mistakenly identified the APT used as the T12 $0.8 \mathrm{~m}$ APT.) Our observing procedures and data reduction procedures in this paper are identical to those described in Howard et al. (2009).

Our three comparison stars A, B, and C for HD 156668 (star D) were HD 158974 ( $V=5.63, B-V=0.96$, G8 III), HD $155092(V=7.07, B-V=0.42, \mathrm{~F} 2)$, and HD 156536 $(V=7.51, B-V=0.42, \mathrm{~F} 3 \mathrm{IV})$, respectively. The T10 APT acquired 477 good observations of this quartet of stars covering the 2007, 2008, and 2009 observing seasons. Comparison star A, HD 158974, exhibits low-amplitude brightness variability of 5 mmag with a period of 152 days. The $C-B$ differential magnitudes have a standard deviation of $1.7 \mathrm{mmag}$, indicating that both stars are constant to the level of our measurement precision. Therefore, we created differential magnitudes of HD 156668 by averaging the $D-B$ and $D-C$ differential magnitudes into a single $D-(B+C) / 2$ differential magnitude to be used for our analyses. We also combined our $b$ and $y$ observations into a single $(b+y) / 2$ passband to improve the precision further.

These $D-(B+C) / 2$ magnitudes in the $(b+y) / 2$ passband are shown in the top panel of Figure 5. The gaps following the longer runs of data are due to the necessity of shutting down APT 
Table 4

Summary of Photometric Observations of HD 156668

\begin{tabular}{lcccc}
\hline \hline $\begin{array}{l}\text { Observing } \\
\text { Season } \\
(1)\end{array}$ & $N_{\text {obs }}$ & $\begin{array}{c}\text { Date Range } \\
(\mathrm{HJD}-2,400,000)\end{array}$ & $\begin{array}{c}\text { Sigma } \\
(\mathrm{mag})\end{array}$ & $\begin{array}{c}\text { Seasonal Mean } \\
(\mathrm{mag})\end{array}$ \\
\hline 2007 & $(2)$ & $(3)$ & $(4)$ & $(5)$ \\
2008 & 181 & $54117-54407$ & 0.00195 & $1.27046 \pm 0.00014$ \\
2009 & 225 & $54484-54754$ & 0.00188 & $1.27121 \pm 0.00013$ \\
\hline
\end{tabular}

operations during the summer rainy season in Arizona. The gaps following the shorter data groups are the normal seasonal gaps for HD 156668. A summary of the photometric observations is given in Table 4. Column 5 indicates that the seasonal mean magnitudes vary over a range of $\sim 1 \mathrm{mmag}$, which is typical for solar-type stars with modest chromospheric activity (see, e.g., Lockwood et al. 2007).

The vertical bars in the top panel of Figure 5 encompass the portion of the $D-(B+C) / 2$ light curve that most clearly exhibits coherent variability that might be due to rotational modulation of spots on the star's photosphere (see, e.g., Henry et al. 1995). That section of the light curve is replotted in the second panel. Straight line segments approximate the 2.5 cycles of the purported brightness variability.

Plotted in the third panel of Figure 5 is the frequency spectrum of the observations from panel two, showing a clear periodicity of 51.5 days. In the bottom panel, the same observations are phased to the 51.5 day period and a time of minimum computed with a least-squares sine fit to the observations. The sine fit also gives a peak-to-peak amplitude of $2.3 \pm 0.3 \mathrm{mmag}$.

We take the 51.5 day period to be the rotation period of HD 156668. This is consistent with $v \sin i=0.5 \mathrm{~km} \mathrm{~s}^{-1}$ and is very close to the rotation period of 48 days predicted from its level of chromospheric activity.

The APT photometry is also useful for confirming that observed RV variations are due to a planetary companion and not stellar surface activity. Queloz et al. (2001) and Paulson et al. (2005) have demonstrated how rotational modulation in the visibility of star spots on active stars can result in periodic RV variations and their misinterpretation. All 477 photometric observations of HD 156668 are plotted in the top panel of Figure 6, phased with the 4.6455 day RV period and a time of mid-transit computed from the orbital elements. A least-squares sine fit on that period gives a semi-amplitude of only $0.10 \pm 0.11 \mathrm{mmag}$. This absence of detectable rotational modulation of surface activity to high precision on the RV period provides strong evidence that the RV variations arise from a super-Earth companion.

Using on the model by Saar \& Donahue (1997), we estimate $\mathrm{RV}$ variability of $\sim 0.8 \mathrm{~m} \mathrm{~s}^{-1}$ (half amplitude) based on $v \sin i$ and the level of photometric variability of this star. Desort et al. (2007) simulated the impact of spots on RV measurements in detail. Scaling from their case of a K2 dwarf (similar to $\mathrm{HD} 156668)$, we estimate $\mathrm{RV}$ variation of $\sim 0.9 \mathrm{~m} \mathrm{~s}^{-1}$ (half amplitude). These estimates are both smaller than, but comparable to, our adopted jitter value of $1.5 \mathrm{~m} \mathrm{~s}^{-1}$, which includes RV noise from other sources.

The bottom panel of Figure 6 replots the photometric observations that lie near the predicted time of transit. The solid curve shows the predicted depth $(3.1 \mathrm{mmag})$ and duration $( \pm 0.011$ phase units) of a central transit, computed from the orbital elements and stellar properties with an assumed planetary composition of pure hydrogen. That such shallow transits
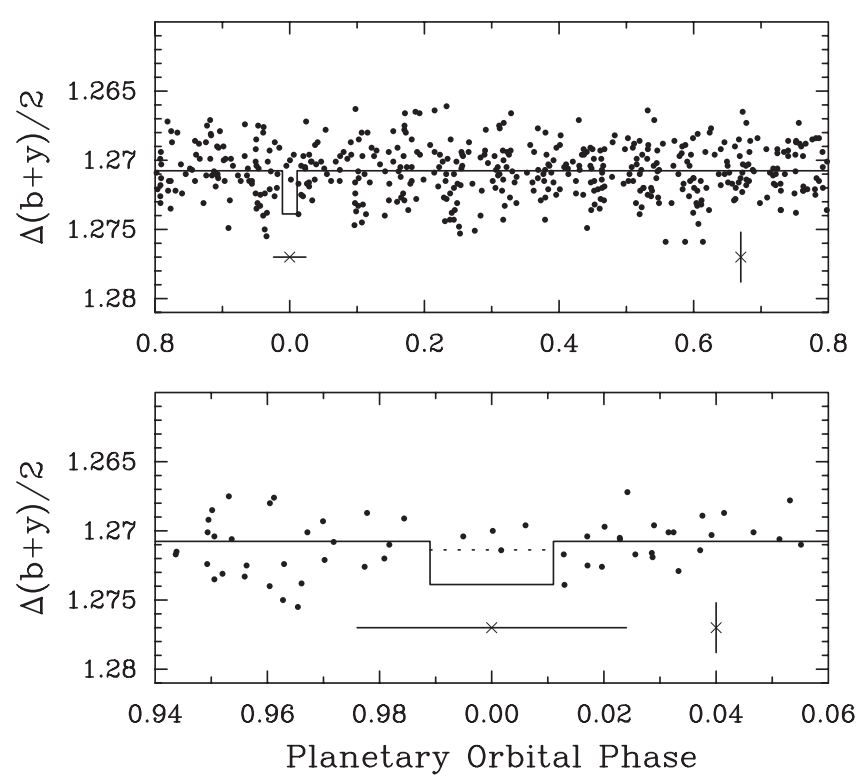

Figure 6. Top panel: the 477 APT photometric observations of HD 156668 plotted modulo the 4.6455 days period of the RV variations. A least-squares sine fit at that period yields a semi-amplitude of only $0.10 \pm 0.11 \mathrm{mmag}$, providing strong evidence that the velocity variations arise from a planetary companion. Bottom panel: the photometric observations of HD 156668 near the predicted time of transit replotted with an expanded scale. The solid curve shows the predicted depth $(3.1 \mathrm{mmag})$ and duration $( \pm 0.011$ phase units $)$ of a central transit, computed from the orbital elements, the stellar properties, and a planetary composition of pure hydrogen. The uncertainty in the predicted transit time is shown by the error bar under the transit window. The dotted line across the transit window shows the expected depth $(0.6 \mathrm{mmag})$ for a planet composed of water. Transits of a pure hydrogen planet are essentially ruled out. Additional observations are required to rule out other planetary compositions.

can be detected with the APTs has been shown by Sato et al. (2005), who used the T11 APT to discover the 3 mmag transits of HD 149026b. The dotted line across the transit window corresponds to the expected depth $(0.6 \mathrm{mag})$ of a hypothetical planet composed of entirely water. Our observations show that transits with a depth of $3.1 \mathrm{mag}$ probably do not occur, essentially ruling out transits of a hydrogen planet. Additional precise photometry is required to rule out other planetary compositions.

\subsection{Chromospheric Activity}

RV planet searches measure the shifts of the centroids of thousands of stellar lines. The shapes of individual lines are determined in large part by Doppler broadening from the disk-averaged velocity field of the star's surface. Spots are magnetically controlled regions of the stellar photosphere characterized by temperatures $\sim 1000 \mathrm{~K}$ lower than unaffected regions. As they rotate across the stellar surface, spots contribute less flux to particular parts of each absorption line profile-less flux on the blue side of lines for spots on the approaching limb and less flux on the red side for spots on the receding limb-thereby distorting the average line profile, shifting the centroid, and causing an apparent Doppler shift of the star.

False positive signals of this type tend to occur around chromospherically active stars (Queloz et al. 2001; Paulson et al. 2005). The spurious RV signals are coherent over typical spot lifetimes (weeks to months) and have periods similar to the stellar rotation period. In Section 5.2, we showed that the 4.6455 day signal is not seen in APT photometry, ruling out rotationally modulated spots as the source of the RV periodicity. Here, we use the chromospheric indices $S_{\mathrm{HK}}$ and $\log R_{\mathrm{HK}}^{\prime}$ to strengthen that conclusion further. These indices measure the 


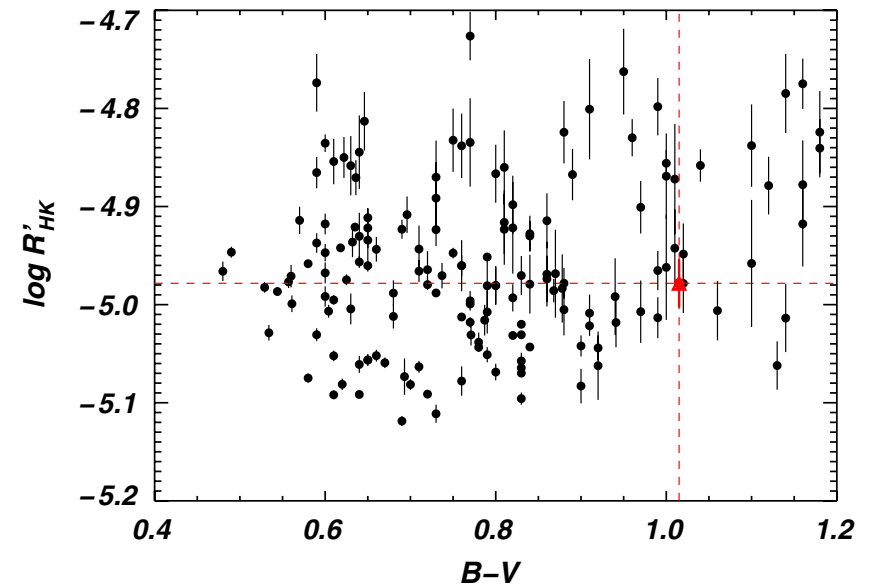

Figure 7. Plot of $\log R_{\mathrm{HK}}^{\prime}$ as a function of $B-V$ color for all $\mathrm{G}$ and $\mathrm{K}$ stars in the Eta-Earth survey with $B-V<1.2$. HD 156668 (large red triangle highlighted by dashed red lines) is among the least chromospherically active Eta-Earth stars with similar $B-V$

level of stellar chromospheric activity, which in turn is strongly correlated with the magnetic activity of the stellar photosphere.

Some stars also show long-term chromospheric activity cycles as the average number of spots rises and falls with the solar cycle, typically with a timescale of $\sim 10$ years. These cycles are sometimes detected as apparent RV shifts and incorrectly interpreted as long-period planets.

Figure 7 shows the average value of $\log R_{\mathrm{HK}}^{\prime}$ for HD 156668 and the other stars in the Eta-Earth Survey with $B-V<1.2$. HD 156668 is among the least chromospherically active stars of similar spectral type in the Eta-Earth Survey. This is consistent with our limits on photometric variability from APT measurements (Section 5.2) and the small RV residuals to the Keplerian fit.

The values of $S_{\mathrm{HK}}$ derived from each Keck-HIRES spectrum are listed in Table 2 and plotted as a time series in Figure 3(d). A Lomb-Scargle periodogram of the data is plotted in Figure 3(h). Importantly, the periodogram shows negligible power for $P \lesssim 20$ days, as expected for a middle-aged star with a predicted rotation period of $\sim 48$ days. This lack of power at periods near 4.6 days strengthens the case for HD $156668 \mathrm{~b}$.

On average, $S_{\mathrm{HK}}$ rises with time (Figure 3(d)), possibly due to the observation of a partial solar magnetic cycle. This several year long trend is not observed in the RVs (Figure 3(a)). One feature of the RV time series is also apparent in the $S_{\mathrm{HK}}$, the declining trend in late 2007. This apparent correlation raises the possibility that the long-period signal that was filtered out of the RVs in Section 4 is due to stellar activity. Despite this apparent correlation, the Pearson linear correlation coefficient, $r=+0.11$, between the unfiltered RVs and the $S_{\mathrm{HK}}$ values indicates an insignificant correlation. When we subtract a second-order polynomial fit to the $S_{\mathrm{HK}}$ values, the correlation with the RVs is still statistically insignificant, $r=+0.19$. Thus, the suggestion that stellar activity explains the longperiod signal is not well supported when the entire data set is considered.

Rotational modulation of spots and other surface features can also be detected in the spectral line bisectors (Torres et al. 2005). However, for this extremely low-amplitude signal $\left(K<2 \mathrm{~m} \mathrm{~s}^{-1}\right)$, detecting line profile variations at the same or higher precision is not possible.

As a final check, we show in Figure 8 that the RV signal from HD $156668 \mathrm{~b}$ is strictly periodic and present throughout

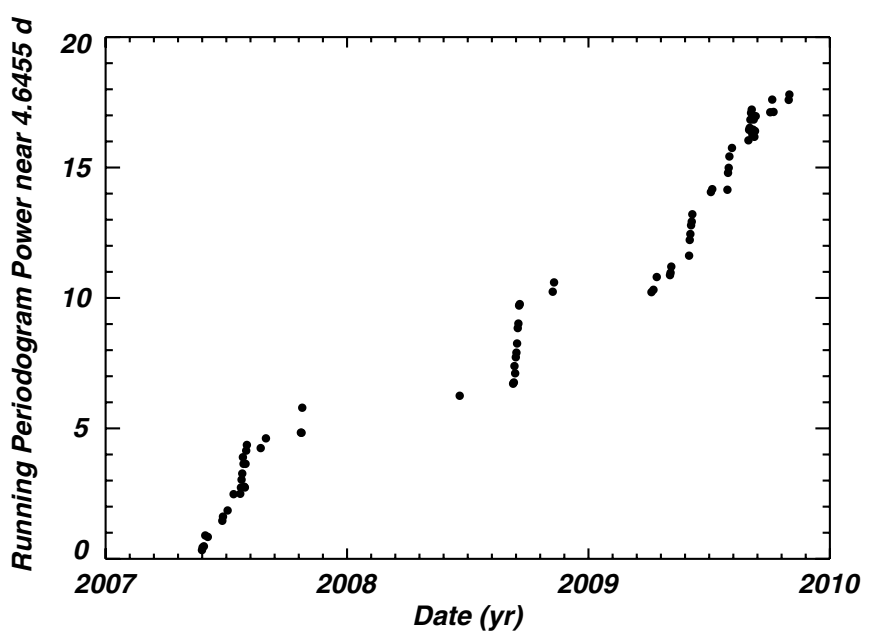

Figure 8. Running periodogram power near $P=4.6455$ days as a function of time. Each dot represents the maximum periodogram power for the set of all high-pass filtered RVs up to that point in time. Only periods near the orbital period of HD $156668 \mathrm{~b}(P=4.64-4.65$ days $)$ were used to compute each maximum periodogram power value. The monotonic rise confirms that the signal was present throughout the observations and points to a dynamical origin.

the observations, as the clock-like signal from a planet should be. The plot shows the periodogram power of the planet rising monotonically as additional measurements are taken. False periodicities, such as those due to spots, typically exhibit periodicities that are only briefly coherent and that may reappear with slightly different periods.

\subsection{Stroboscopic Alias}

We interpret the periodogram spike near 1.270 days (Figure 3) as a stroboscopic alias of the orbital period (which appears on the periodogram as 4.647 days due to finite period sampling) with the sidereal day length (0.997 days): 1/4.647 days + $1 / 1.270$ days $=1 / 0.997$ days. ${ }^{11}$ These two periods have nearly equal periodogram amplitudes in our measurements and in simulated data sets with synthetic signals of either period injected. Nevertheless, we interpret the peak at 4.647 days as the physical period because of the substantially greater abundance of planets with such orbital periods. For comparison, we consider the initial data release from the Kepler spacecraft which contains 312 planet candidates (Borucki 2010). Of those, 21 planet candidates have periods in the range $P=4-8$ days and planet radii of $<2.5 R_{\oplus}$ (super-Earths and smaller). Kepler has detected zero planet candidates with $P=1-2$ days (the same range of $\log P$ ) and planet radii of $<2.5 R_{\oplus}$. Note that Kepler is highly sensitive to short-period planets, and does not suffer from aliasing, because of fixed pointing from space and high photometric precision.

\section{DISCUSSION}

We present the detection of HD $156668 \mathrm{~b}$, a super-Earth planet with a minimum mass of $M_{P} \sin i=4.15 M_{\oplus}$ in a $P=4.6455$ day orbit around a K3 dwarf. We draw on several

\footnotetext{
11 After the submission of our manuscript, Dawson \& Fabrycky (2010) claimed that a single-planet model with a physical period of $P=1.270$ days is preferred over a single-planet model with a physical period of $P=4.647$ days. Their analysis is based on a study of the spectral window function and the relative strength of alias periods, but does not model the long-period signal that we include.
} 
lines of evidence to support the existence of HD $156668 \mathrm{~b}$. We showed in Section 5.1 that the short-period signal is statistically significant. This signal is apparent in a fit to the unfiltered RVs and stands out strongly when isolated by high-pass filtering. The host star is middle-aged and quiet, providing a nearly ideal RV target. The planet's short-period signature is not seen in photometric observations or in chromospheric indices. Thus, the evidence strongly points to a planetary interpretation.

To estimate the orbital parameters of HD $156668 \mathrm{~b}$, we found it necessary to apply a high-pass filter to the RVs by subtracting the model of a long-period Keplerian. This model changes slowly over time and clarifies, but does not artificially enhance, the $P=4.6455$ day signal of HD 156668 b. Filtering of this type is common in other areas of time domain astronomy (e.g., transit photometry, astroseismology) and is uncommon, but not unprecedented, in RV planet detection. Similar filtering techniques were employed to disentangle the planetary signals of Gl 176 b (Forveille et al. 2009) and Gl 674 b (Bonfils et al. 2007), although in those cases the non-planetary signal was clearly due to spots modulated by stellar rotation. Queloz et al. (2009) used a "pre-whitening" technique to extract the signatures of two super-Earths from the complicated RV time series of the significantly more active star, Corot-7. Further, many exoplanets are announced with a model that includes a linear or second-order RV trend, presumably due to a longperiod orbital companion. The source of the long-period signal remains unknown and motivates additional measurements. If it is due to a planet, the body has minimum mass $\sim 45 M_{\oplus}$ and orbits with $P_{c}=2.31$ years and $a_{c}=1.6 \mathrm{AU}$, a cold superNeptune near the ice line.

We see no evidence for transits of HD $156668 \mathrm{~b}$ down to a level of $\sim 3$ mmag. However, given the large a priori transit probability of $7 \%$, it is instructive to speculate about the transit signatures of various possible planet compositions. Using the models in Seager et al. (2007), a $4 M_{\oplus}$ planet composed of pure Fe, $\mathrm{MgSiO}_{3}, \mathrm{H}_{2} \mathrm{O}$, or $\mathrm{H}$ would yield planets of radius $R_{\mathrm{pl}}=1.2$, $1.5,2.0$, and $4.5 R_{\oplus}$, producing transits of depth $0.22,0.35,0.61$, and $3.1 \mathrm{mmag}$, respectively. These homogeneous planet models are oversimplified, but set the scale for admixtures of those ingredients: transits of planets made of solids and water would have depths of $\sim 0.2-0.6 \mathrm{mmag}$, while transits of a planet with a significant atmosphere could be much deeper. For comparison, the transiting super-Earth Corot-7 b has a transit depth of 0.36 mmag implying a radius $R_{\mathrm{pl}}=1.68 R_{\oplus}$ (Léger et al. 2009). Using the RV-determined mass $M_{\mathrm{pl}}=4.8 M_{\oplus}$ (Queloz et al. 2009), the bulk density is terrestrial, $\rho_{\mathrm{pl}}=5.6 \mathrm{~g} \mathrm{~cm}^{-3}$. In contrast, GJ $1214 \mathrm{~b}$ has $M_{\mathrm{pl}}=6.6 M_{\oplus}$ and $R_{\mathrm{pl}}=2.7 R_{\oplus}$, implying $\rho_{\mathrm{pl}}=1.9 \mathrm{~g} \mathrm{~cm}^{-3}$, intermediate between Earth and the ice giants of the solar system. Transits of GJ $1214 \mathrm{~b}$ are unusually deep (15 mmag) for a planet of this size because it orbits an M dwarf with $R_{\star}=0.21 R_{\odot}$ (Charbonneau et al. 2009).

Several authors (e.g., Ida \& Lin 2004, Mordasini et al. 2009) have argued that super-Earths will have insignificant hydrogen atmospheres (by mass) because during formation their masses failed to reach a critical mass (typically $\sim 10 M_{\oplus}$ ) when the growth from solid planetesimals is augmented by runaway accretion of gas from the protoplanetary disk. Smaller atmospheres (up to several percent by mass) could be produced by degassing during impact accretion and geological activity (Elkins-Tanton \& Seager 2008; Kite et al. 2009). However, whatever atmosphere is acquired from these processes may be lost to atmospheric escape (Baraffe et al. 2006; Valencia et al. 2010). Nevertheless, the brief history of exoplanets is replete with observational surprises (hot Jupiters, eccentric orbits, etc.) so we consider the observational consequences of an atmosphere. Adams et al. (2008) find that adding an $\mathrm{H} / \mathrm{He}$ gas envelope equivalent to $0.2 \%-20 \%$ of the mass of a solid $5 M_{\oplus}$ exoplanet increases the radius $8 \%-110 \%$ above the gas-free value. Atmospheres dominated by heavier molecules such as $\mathrm{H}_{2} \mathrm{O}$ and $\mathrm{N}_{2}$ (as on Earth) would swell the planet less for the same atmospheric mass because of the higher mean molecular weight and reduced scale height. Thus, we conclude that the APT photometric observations rule out transits for HD $156668 \mathrm{~b}$ if the radius is dominated by an $\mathrm{H} / \mathrm{He}$ atmosphere (tens of percent by mass), but do not preclude transits if the atmosphere is less massive or composed of heavier elements.

HD $156668 \mathrm{~b}$ adds statistical weight to the emerging trends of the properties of super-Earths and their hosts. Like most other such planets, it orbits a K or M dwarf. In contrast to other superEarth hosts, HD 156668 has a slightly super-solar metallicity (Howard et al. 2009). The rate of multiplicity in systems with super-Earths and Neptune-mass planets appears to be much higher than for higher mass planet hosts, with HD 40307 (Mayor et al. 2009a), GJ 581 (Mayor et al. 2009b), and HD 69830 (Lovis et al. 2006) being the standard examples of multiplicity in lowmass systems. The long-period signal seen for HD 156668 is suggestive of a second low-mass planet in the system.

HD $156668 \mathrm{~b}$ pushes the frontier of RV planet discovery to lower masses and smaller Doppler amplitudes. It is the second lowest minimum mass exoplanet discovered to date by the RV technique, after GJ 581 e (Mayor et al. 2009b). With a Doppler semi-amplitude of $1.89 \mathrm{~m} \mathrm{~s}^{-1}$, HD $156668 \mathrm{~b}$ is also only the second exoplanet discovered to date with $K<2.0 \mathrm{~m} \mathrm{~s}^{-1}$. GJ $581 \mathrm{e}$ is the other with $K=1.85 \mathrm{~m} \mathrm{~s}^{-1}$ and $M \sin i=1.9 M_{\oplus}$ (Mayor et al. 2009b). This progress is remarkable: 51 Peg b was discovered (Mayor \& Queloz 1995) with $M \sin i=0.47 M_{\mathrm{JUP}}$ and $K=57 \mathrm{~m} \mathrm{~s}^{-1}$ while the signal from HD $156668 \mathrm{~b}$ is smaller by factors of 35 and 30 , respectively.

There is significant disagreement within the Doppler community over the ultimate limits of RV detectability. In our view, true Earth analogs $-1 M_{\oplus}$ planets in $1 \mathrm{AU}$ orbits around G stars-will remain permanently out of reach of the Doppler technique and will instead be discovered by transit photometry, astrometry, and microlensing. The Earth imparts a $K=0.09 \mathrm{~m} \mathrm{~s}^{-1}$ signal on the Sun, a factor of 20 smaller than HD 156668 b. Detecting such a signal requires roughly $20^{2}=4000$ times as many photons. If this improvement is ever achieved, it will be by a combination of observing brighter stars with larger telescopes and more efficient spectrometers. A further complication is stellar jitter, which in our view likely limits Doppler precision to a few tenths of a $\mathrm{m} \mathrm{s}^{-1}$ for the most quiet stars, even when averaged over with long integrations. Longer period planets suffer from the additional disadvantage that only a few orbits transpire during an observational campaign (compared with $\sim 100$ orbits for the discoveries of HD $155668 \mathrm{~b}$ and GJ $581 \mathrm{e}$ ), reducing their detectability by clock-like coherence.

Nevertheless, the future of the Doppler technique is bright and we expect that it will continue to play a prominent role in planet searches. Discoveries of super-Earths and Neptunes from the Eta-Earth Survey will shape our understanding of planet formation and migration. Doppler work in other domains-long period giant planets, subgiants, multi-planet systems, dynamically interacting planets, Rossiter-McLaughlin measurements, etc.-will also continue to inform and enrich our knowledge of the rich astrophysics of exoplanets. 
We thank the many observers who contributed to the velocities reported here. We gratefully acknowledge the efforts and dedication of the Keck Observatory staff, especially Scott Dahm, Hien Tran, and Grant Hill for support of HIRES and Greg Wirth for support of remote observing. We thank Heather Knutson, Doug Lin, Shigeru Ida, Jeff Scargle, and Ian Howard for helpful discussions. We are grateful to the time assignment committees of the University of California, NASA, and NOAO for their generous allocations of observing time. Without their long-term commitment to RV monitoring, these long-period planets would likely remain unknown. We acknowledge R. Paul Butler and S. S. Vogt for many years of contributing to the data presented here. A.W.H. gratefully acknowledges support from a Townes Post-doctoral Fellowship at the U. C. Berkeley Space Sciences Laboratory. G.W.M. acknowledges the NASA Grant NNX06AH52G. G.W.H. acknowledges support from NASA, NSF, Tennessee State University, and the State of Tennessee through its Centers of Excellence program. J.T.W. was partially supported by funding from the Center for Exoplanets and Habitable Worlds. The Center for Exoplanets and Habitable Worlds is supported by the Pennsylvania State University, the Eberly College of Science, and the Pennsylvania Space Grant Consortium. This work made use of the SIMBAD database (operated at CDS, Strasbourg, France), NASA's Astrophysics Data System Bibliographic Services, and the NASA Star and Exoplanet Database (NStED). Finally, the authors wish to extend special thanks to those of Hawai'ian ancestry on whose sacred mountain of Mauna Kea we are privileged to be guests. Without their generous hospitality, the Keck observations presented herein would not have been possible.

\section{REFERENCES}

Adams, E. R., Seager, S., \& Elkins-Tanton, L. 2008, ApJ, 673, 1160

Bakos, G. Á., et al. 2010, ApJ, 710, 1724

Baraffe, I., Alibert, Y., Chabrier, G., \& Benz, W. 2006, A\&A, 450, 1221

Beaulieu, J., et al. 2006, Nature, 439, 437

Bennett, D. P., et al. 2008, ApJ, 684, 663

Bonfils, X., et al. 2007, A\&A, 474, 293

Borucki, W. J. 2010, arXiv:1006.2799

Borucki, W. J., et al. 2009, Science, 325, 709

Butler, R. P., Marcy, G. W., Williams, E., McCarthy, C., Dosanjh, P., \& Vogt, S. S. 1996, PASP, 108, 500

Charbonneau, D., et al. 2009, Nature, 462, 891

Cumming, A. 2004, MNRAS, 354, 1165
Dawson, R. I., \& Fabrycky, D. C. 2010, ApJ, 722, 937

Demarque, P., Woo, J., Kim, Y., \& Yi, S. K. 2004, ApJS, 155, 667

Desort, M., Lagrange, A., Galland, F., Udry, S., \& Mayor, M. 2007, A\&A, 473, 983

Elkins-Tanton, L. T., \& Seager, S. 2008, ApJ, 685, 1237

Forveille, T., et al. 2009, A\&A, 493, 645

Gray, R. O., Corbally, C. J., Garrison, R. F., McFadden, M. T., \& Robinson, P. E. 2003, AJ, 126, 2048

Henry, G. W., Fekel, F. C., \& Hall, D. S. 1995, AJ, 110, 2926

Høg, E., et al. 2000, A\&A, 355, L27

Howard, A. W., et al. 2010, ApJ, 721, 1467

Howard, A. W., et al. 2009, ApJ, 696, 75

Ida, S., \& Lin, D. N. C. 2004, ApJ, 604, 388

Isaacson, H. T., \& Fischer, D. A. 2010, ApJ, in press (arXiv:1009.2301)

Jansson, P. 1995, Deconvolution: With Applications in Spectroscopy (New York: Academic)

Kite, E. S., Manga, M., \& Gaidos, E. 2009, ApJ, 700, 1732

Léger, A., et al. 2009, A\&A, 506, 287

Lockwood, G. W., Skiff, B. A., Henry, G. W., Henry, S., Radick, R. R., Baliunas, S. L., Donahue, R. A., \& Soon, W. 2007, ApJS, 171, 260

Lomb, N. R. 1976, Ap\&SS, 39, 447

Lovis, C., et al. 2006, Nature, 441, 305

Marcy, G. W., \& Butler, R. P. 1992, PASP, 104, 270

Marcy, G. W., Butler, R. P., Vogt, S. S., Fischer, D. A., Henry, G. W., Laughlin, G., Wright, J. T., \& Johnson, J. A. 2005, ApJ, 619, 570

Mayor, M., \& Queloz, D. 1995, Nature, 378, 355

Mayor, M., et al. 2009a, A\&A, 493, 639

Mayor, M., et al. 2009b, A\&A, 507, 487

Mordasini, C., Alibert, Y., \& Benz, W. 2009, A\&A, 501, 1139

Noyes, R. W., Hartmann, L. W., Baliunas, S. L., Duncan, D. K., \& Vaughan, A. H. 1984, ApJ, 279, 763

O’Toole, S., et al. 2009, ApJ, 697, 1263

Paulson, D. B., Sneden, C., \& Cochran, W. D. 2005, AJ, 130, 2436

Queloz, D., et al. 2001, A\&A, 379, 279

Queloz, D., et al. 2009, A\&A, 506, 303

Saar, S. H., Butler, R. P., \& Marcy, G. W. 1998, ApJ, 498, L153

Saar, S. H., \& Donahue, R. A. 1997, ApJ, 485, 319

Sato, B., et al. 2005, ApJ, 633, 465

Scargle, J. D. 1982, ApJ, 263, 835

Seager, S., Kuchner, M., Hier-Majumder, C. A., \& Militzer, B. 2007, ApJ, 669, 1279

Sumi, T., et al. 2010, ApJ, 710, 1641

Torres, G., Konacki, M., Sasselov, D. D., \& Jha, S. 2005, ApJ, 619, 558

Unwin, S. C., et al. 2008, PASP, 120, 38

Valencia, D., Ikoma, M., Guillot, T., \& Nettelmann, N. 2010, A\&A, 516, 20

Valenti, J. A., Butler, R. P., \& Marcy, G. W. 1995, PASP, 107, 966

Valenti, J. A., \& Fischer, D. A. 2005, ApJS, 159, 141

Valenti, J. A., et al. 2009, ApJ, 702, 989

van Leeuwen, F. 2007, A\&A, 474, 653

Vogt, S. S., et al. 1994, Proc. SPIE, 2198, 362

Wright, J. T. 2005, PASP, 117, 657

Wright, J. T., \& Howard, A. W. 2009, ApJS, 182, 205

Wright, J. T., Marcy, G. W., Butler, R. P., \& Vogt, S. S. 2004, ApJS, 152, 261 